\title{
A Case Study of Puerperal Group A Streptococcal Infection Complicated by Toxic Shock Syndrome
}

\author{
Robert J. Olp, MD ${ }^{1}$ Ingrid A. Chamales, MD ${ }^{1}$ Stacey S. Schmiedecke, MD, LCDR, MC, USN ${ }^{10}$ \\ ${ }^{1}$ Department of Obstetrics and Gynecology, Madigan Army Medical \\ Center, Tacoma, Washington \\ 2 Department of Maternal Fetal Medicine, Madigan Army Medicel \\ Center, Tacoma, Washington

\begin{abstract}
Address for correspondence Stacey S. Schmiedecke, MD, LCDR, MC, USN, Department of Maternal Fetal Medicine, Madigan Army Medicel Center, 9040 Jackson Avenue, Tacoma, WA 98431 (e-mail: s13gator@gmail.com).
\end{abstract}

Am J Perinatol Rep 2020;10:e1-e4.

\begin{abstract}
Keywords

- group A streptococcus

- toxic shock syndrome

- puerperal infection

- sepsis

- Streptococcus pyogenes

Puerperal infection with group A streptococcus (GAS), Streptococcus pyogenes, is associated significant morbidity and mortality. When associated with toxic shock syndrome (TSS), mortality rates rise to approximately $50 \%$. We present the case of a 32year-old Para 2 reporting severe left distal lower extremity pain, fevers, and chills at 1 week following an uncomplicated vaginal delivery. The patient's clinical status rapidly decompensated to septic shock requiring transfer to the intensive care unit. She underwent anterior and lateral compartment fasciotomy of the left lower extremity for concerns of possible necrotizing soft tissue infection. Final blood cultures confirmed GAS infection with unclear primary source, though endometritis was favored. She required additional orthopaedic procedures including an arthroscopy with washout for contralateral septic arthritis and myositis before her discharge on hospital day 19 . She obtained a near-full recovery complicated by poor wound healing and permanent left foot drop. While GAS remains a rare puerperal event, obstetricians should recall there is a 20 -fold increased incidence among postpartum women. Progression to TSS is associated with very poor prognosis and hysterectomy is often necessary. Favorable outcomes in GAS with or without TTS hinge on astute clinical suspicion, aggressive fluid resuscitation, early antibiotic therapy, and source control.
\end{abstract}

Group A streptococcus (GAS), Streptococcus pyogenes, remains a highly communicable infection. Transmission occurs from person-to-person, through respiratory droplet spread, or contact with secretions, and has been reported in both community and nosocomial settings. GAS is rarely present in the normal vaginal flora and can be rapidly fatal in the setting of a pregnant or recently pregnant woman. With the onset of regular hand washing by physicians, first proposed by Austrian physician Ignaz Semmelweis in 1847, rates of puerperal GAS sepsis have declined significantly. ${ }^{1}$ Noninvasive GAS includes cellulitis and accounts for approximately $40 \%$ of GAS cases. The remainder of cases are classified as invasive GAS, describing systemic sequelae, such as instance primary bacteremia, necrotizing fasciitis,

received

May 19, 2019

accepted

May 25, 2019
DOI https://doi.org/

10.1055/s-0039-1697648. ISSN 2157-6998. pneumonia, and streptococcal toxic shock syndrome (TSS). ${ }^{2-7}$ Invasive postpartum GAS infection produces symptoms usually within 2 to 3 days following delivery. High fever and chills from resultant bacteremia are common. While the patient may not present with severe uterine tenderness, endometritis is a common etiology. Specifically, over $75 \%$ of postpartum women with invasive GAS have either endometritis or infection without identifiable focus. ${ }^{5}$ Wound infection can occur following vaginal or Cesarean delivery or other abdominal surgeries (e.g., postpartum tubal ligation) with development of systemic complications if not promptly identified and treated.

Sepsis caused by GAS can progress to shock and multiorgan failure within 48 to 96 hours. $^{8}$ This is referred to as
Copyright $\odot 2020$ by Thieme Medical Publishers, Inc., 333 Seventh Avenue, New York, NY 10001, USA. Tel: +1(212) 760-0888.
License terms

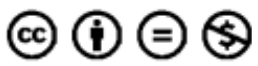


streptococcal TSS and affects approximately $6 \%$ of adults with invasive GAS. The mortality rate of GAS TSS is devastatingly high at 40 to $60 \%{ }^{7}$ Symptoms vary and may include endometritis, wound cellulitis, necrotizing fasciitis, or pneumonia among others. More commonly, however, the patient will report nonspecific symptoms. Admission to a critical care unit is necessary for supportive measures, with initiation of aggressive fluid resuscitation, antibiotic therapy, and primary source control. Early surgical intervention is often necessary without waiting for response to antibiotics. In the case of postpartum invasive GAS, hysterectomy is frequently recommended for adequate source control. We describe a case of postpartum invasive GAS complicated by TTS in which rapid recognition and treatment resulted in maternal survival without need for hysterectomy but not without significant morbidity.

\section{Case Presentation}

A 32-year-old Gravida 2 Para 2 presented on postpartum day 7 following an uncomplicated late-term vaginal delivery. She reported fevers, chills, and left distal lower extremity pain and swelling, as well as swelling in the right knee. She denied recent injury to the affected limbs, but was unable to bear weight on the left side. She also reported a diffuse rash involving her upper abdomen. She denied other postpartum complications and had continued breastfeeding her infant. Her past medical history was notable for history of stage IIa malignant peripheral nerve sheath tumor in remission that was treated 1-year prior by complete surgical excision without chemotherapy.

As for her recent delivery course, the patient was admitted in labor at $42^{0 / 7}$ weeks gestation. Her antepartum course was uncomplicated and group B streptococcus carrier screening was negative. Small labial and first-degree midline lacerations were encountered and repaired at time of delivery. Minimal blood loss was noted. She had an unremarkable postpartum course and was discharged home on postpartum day 1 with her newborn. Upon presentation 6 days later, she reported fevers to $104^{\circ} \mathrm{F}\left(40^{\circ} \mathrm{C}\right)$ at home. Her initial vital signs included a temperature $(\mathrm{T})$ of $97.5^{\circ} \mathrm{F}\left(36.4^{\circ} \mathrm{C}\right)$, heart rate $(\mathrm{HR})$ of 68 beats per minute (bpm), respiratory rate (RR) of 18 , and blood pressure (BP) of 107/69. She appeared in mild distress, endorsing moderate, "tight-like" pain to her left distal lower extremity. Examination was remarkable for limited range of motion of the left lower extremity and a focal area of erythema measuring approximately $2 \mathrm{~cm} \times 3 \mathrm{~cm}$ at her left ankle (-Fig. 1). Additionally, the affected extremity was exquisitely tender to palpation along the length of the anterior aspect of the left tibia. Her right knee appeared swollen, and prominently asymmetric in size compared with the left knee. Peripheral pulses were present bilaterally. Her abdomen was nonperitoneal but her uterus was documented to be mildly tender on bimanual examination with fundus firm at $4-\mathrm{cm}$ below the umbilicus. A faint maculopapular rash was noted extending to the inferior edge of her breasts. Scant nonpurulent lochia was noted on pelvic examination. Laboratory findings were notable for a leukocytosis to

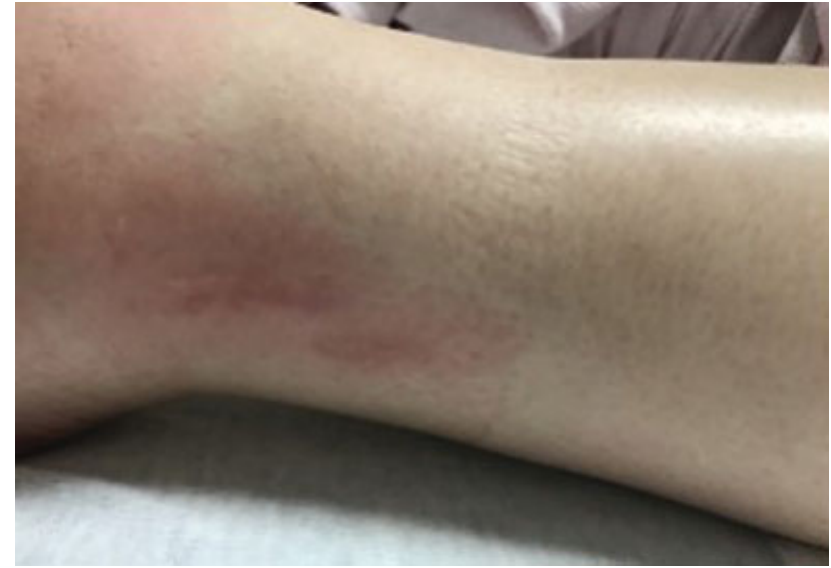

Fig. 1 Solitary physical exam finding of focal left ankle erythema in the setting of severe distal left lower extremity pain.

18,800 , with a bandemia of $23.7 \%$ and lactate of $1.6 \%$. A recent clean-catch urine culture was notable for $>100,000$ colony-forming units per high-power field (CFU/HPF) of S. pyogenes isolate. Orthopaedics was consulted for further evaluation with concern for possible compartment syndrome with concerns for underlying GAS necrotizing infection. Plain X-ray films demonstrated soft tissue edema overlying the anterior left tibia and foot. No osseous abnormalities were noted. The Doppler ultrasonography was obtained and negative for deep vein thrombosis. A left ankle arthrocentesis was performed with reportedly normal appearing fluid obtained. At this time, approximately 2 hours following presentation, the patient's vital signs were notable for $\mathrm{HR}=144 \mathrm{bpm}, \mathrm{T}=102.6^{\circ} \mathrm{F}\left(39.2^{\circ} \mathrm{C}\right), \mathrm{RR}=20$, Mean Arterial Pressure $(\mathrm{MAP})=66$, and oxygen saturation $\left(\mathrm{SpO}_{2}\right)$ $=98 \%$. Aggressive fluid resuscitation was initiated and blood cultures obtained. Intravenous (IV) amoxicillin-sulbactam and clindamycin were started given suspicion for possible invasive GAS infection. Due to concern for TTS, urgent transfer to the intensive care unit (ICU) was undertaken. Computed tomography (CT) of the left lower extremity noted severe inflammation without evidence of abscess or findings suggestive of necrotizing fasciitis. Given the severity of patient's pain, she underwent a left distal lower extremity anterior and lateral compartment fasciotomy (-Fig. 2). No purulence or necrotic tissue was noted at the time of

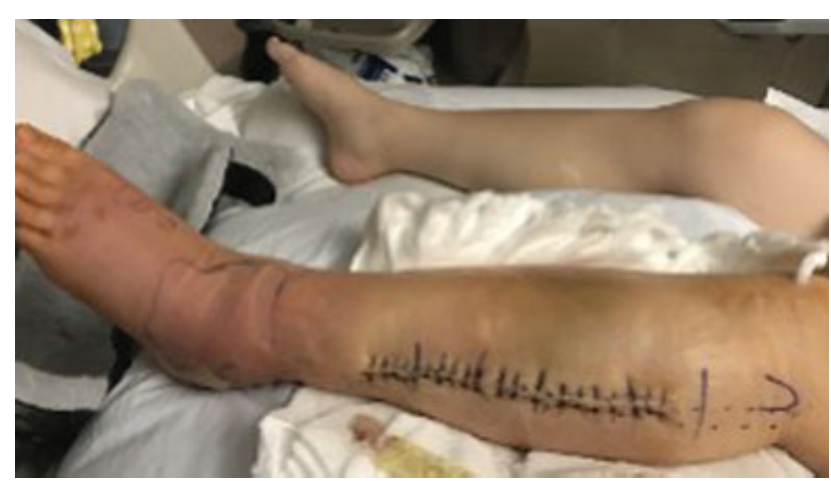

Fig. 2 Status after left distal lower extremity anterior and lateral compartment fasciotomy. 
operation, with normal appearing fascia within an edematous lateral compartment. The patient noted improvement in pain following surgery. She received albumin, norepinephrine, and esmolol briefly for pressure support and control of tachycardia postoperatively. Additionally, she received gentamicin for 48 hours for endometritis coverage given the unclear source of her evolving infection and her recent postpartum state with tender uterus at time of initial evaluation. She also received therapeutic enoxaparin ( $1 \mathrm{mg} / \mathrm{kg}$ twice daily) for possible septic pelvic thrombophlebitis. Her tachycardia, fevers, and leukocytosis resolved and she was stable for transfer out of the ICU on hospital day 5.

Her hospital course following transfer out of the ICU was notable for residual peroneal nerve palsy secondary to her initial fasciotomy. She also required reoperation of the left distal lower extremity for concerns of myositis on hospital day 8. A small area of purulence and decreased contractility of the anterior compartment was noted at that time, with no other abnormalities in color, consistency, or perfusion. On hospital day 13 , she experienced a fever to $102.2^{\circ} \mathrm{F}\left(39^{\circ} \mathrm{C}\right)$ with new leukocytosis. At that time, the patient endorsed worsening pain in her right knee, and an arthrocentesis was performed, productive of frank purulent fluid. She subsequently underwent a right knee arthroscopy, synovectomy, and debridement for right knee septic arthritis. The procedure noted inflamed proliferative synovium throughout the entirety of the knee. She underwent repeat right knee arthroscopic incision and drainage 2 days later on hospital day 15 with negative culture. She was transitioned to IV penicillin G 20 million units/day on hospital day 17. She ultimately was discharged to home on hospitalday 19 , with continued treatment with IV penicillin for a total of 14 days followed by an additional 14 days of oral amoxicillin $500 \mathrm{mg}$ thrice daily. While the primary source of infection of this patient's GAS was not definitely determined, endometritis was suspected as this is a common puerperal etiology and is often largely asymptomatic at time of presentation, as in our patient who had only mild uterine tenderness at time of initial examination and nonpurulent lochia. Cystitis (based on recent GAS positive urine culture) was felt to be less likely as this was a clean-catch specimen and could have represented contamination with vaginal fluid.

The patient continued to work with physical and occupational therapy and supportive boot for her persistent residual peroneal palsy of her left lower extremity. Follow-up visits with orthopaedics noted no evidence of osteomyelitis or continued infection; however, chondromalacia of her left patella was diagnosed which was suspected to have contributed to her ongoing pain, as well as diminished range of motion, and poor flexion during her postoperative recovery. She continued to suffer from poor wound healing at site of her left lower extremity fasciotomy, despite close follow-up and in-office treatments. She underwent further consultation with outside orthopaedic facility for ongoing care. The patient was also noted to have multiple enlarged inguinal lymph nodes, with enlargement on ultrasound redemonstrated 3 weeks later following initial discovery. Her marked lymphadenopathy was suspected to be secondary to a reactive process from these ongoing wound issues in the left lower extremity. Given her history of malignant peripheral nerve sheath tumor, a left inguinal lymph node excisional biopsy, and left lower leg wound debridement were performed almost 2 months following her hospital discharge. Her lymph node biopsy was negative for metastatic tumor, with findings consistent with reactive follicular hyperplasia. Final blood cultures and infectious laboratories confirmed return to baseline and resolution of her GAS infection. She continues to undergo orthopaedic care, as well as physical and occupational therapy, for management of her residual deficits.

\section{Discussion}

Although an infrequent cause of infection, invasive GAS is associated with a significant mortality rate at 15 to $20 \%$. The Centers for Disease Control and Prevention (CDC) estimates that approximately 11,000 to 13,000 cases of invasive GAS disease occur each year in the United States, accounting for approximately 1,600 deaths annually. ${ }^{1}$ Postpartum women have a 20 -fold increased incidence of invasive GAS compared with nonpregnant women with an estimated 200 cases occurring annually in the United States, at a rate of 6 per 100,000 live births. ${ }^{1}$ The increased risk of streptococcal disease in the puerperium is likely related to changes in host immunity that allow for spread to invasive GAS. Theories include damage to cutaneous barriers during delivery, altered vaginal $\mathrm{pH}$ from amniotic fluid exposure, or altered innate immunity related to pregnancy. ${ }^{7}$

Streptococcal TSS complicates approximately $6 \%$ of invasive GAS in adults. Massive inflammatory response occurs secondary to antigen release by host lymphocytes. This is associated with a case fatality rate of approximately $50 \%{ }^{6}$ Presenting symptoms in postpartum patients vary and can include endometritis, wound cellulitis or vulvar infection but are most often nonspecific. Bacteremia is common, as is systemic organ dysfunction and marked bandemia (greater than $10 \%$ ) even in the absence of leukocytosis. Providers should recall that pregnancy causes alterations in serum laboratory parameters. For example, a creatinine $>1 \mathrm{mg} / \mathrm{dL}$ may signal renal impairment in a pregnant or postpartum patient. ${ }^{7}$ If fever is not present, the nonspecific nature of presenting symptoms may delay diagnosis and increase the risk of complications developing. In the case of our postpartum patient, fever and tachycardia with severe left lower extremity pain elevated concerns for evolving sepsis, leading to prompt supportive measures and surgical evaluation. Source control is vital and may require abscess drainage or hysterectomy. In cases of TSS, admission to an ICU is usually required for supportive care, as in the case of this patient.

The suspected source of infection for this patient was endometritis but is ultimately unknown as is the case for many postpartum invasive GAS cases. Postpartum GAS-related endometritis occurs from ascension of bacteria into the uterus and peritoneal cavity. Patients typically have no significant risk factors, for example, prolonged rupture of membranes or chorioamnionitis. Signs and symptoms of GAS endometritis can be mild and nonspecific, including fever, chills, nausea, 
vomiting, and myalgias. ${ }^{8}$ Uterine or pelvic tenderness may not be significant, and subsequently poorly correlate with the severity of the infection. Blood cultures are important to obtain before initiation of antibiotics as long this does not cause delay in patient treatment. GAS can still produce toxins that can lead toTSS and further tissue necrosis. In such cases, hysterectomy is nearly always required to ensure complete removal of the toxin source. ${ }^{1}$

The Surviving Sepsis Campaign, in 2013, discussed four major tenets of management of the septic patient. ${ }^{9}$ First, initial fluid resuscitation to aid in tissue perfusion is critical. Initial fluid resuscitation should be accomplished with 30 $\mathrm{mL} / \mathrm{kg}$ of crystalloid fluids within 3 hours of presentation. ${ }^{7}$ Next step was searching for a diagnosis and initiating early antimicrobial therapy. A differential can be accomplished by detailed history and physical, directed imaging, and culture collection to identify the offending micro-organism. Antibiotic treatment for GAS should cover both $S$. pyogenes and $S$. aureus and may include penicillin or cephalosporin in combination with vancomycin for S. aureas overage and clindamycin as it helps decrease GAS colony counts even when penicillin has reached its peak effect by decreasing toxin production. If the uterus is a suspected source, an aminoglycoside is often added and gram stain and culture can be obtained via endometrial biopsy in the postpartum patient. If the gestation is ongoing, neonatal consequences may arise, and can lead to indication for delivery. Typically GAS is associated with chorioamnionitis in the setting of intact membranes and can be diagnosed via amniocentesis. ${ }^{7}$ Finally, source control within the first 12 hours of presentation is critical to improve outcomes. ${ }^{9}$ Long-term antibiotic therapy is necessary and our specific patient required a total of almost 5 weeks of IV antibiotic therapy followed by 2 weeks of an oral regimen. Hysterectomy is frequently recommended and required in the management in GAS puerperal sepsis due to high-mortality rate, especially in patient deteriorating with organ failure. ${ }^{7}$ Source control remained a difficult task with our patient, as no focus of infection was definitively localized. Given the patient's recent delivery and uterine tenderness, albeit mild, documented at time of admission, endometritis was suspected and, fortunately, hysterectomy was not required. Given her positive recent urine culture, cystitis as a primary source remains possible, though it is important to note this was not a catheterized specimen and may have represented a vaginal culture if the clean-catch was not appropriately collected by the patient.

If survival of invasive GAS TTS can be achieved, physicians and patients must be prepared to address the long-term sequelae of such a devastating event. These include renal impairment, possible need for amputation of affected limb, as well as other potential consequences from debridement. In our case, the patient suffered foot drop and poor-wound healing related to her multiple orthopaedic surgeries, but did not require amputation of the left lower extremity. She continues to require physical and occupational therapy for deficits encountered postoperatively.

While a rare puerperal event, obstetricians should not forget the increased risk of developing invasive GAS in the puerperium. Morbidity and mortality remain extremely high, particularly, if there is development of TTS. Favorable outcomes, as in the case of our patient, are dependent upon high-clinical suspicion, early diagnosis, and initiation treatment with aggressive fluid resuscitation, antibiotic therapy, and source control.

\section{Conflict of Interest}

None.

\section{References}

1 Rimawi BH, Soper DE, Eschenbach DA. Group A streptococcal infections in obstetrics and gynecology. Clin Obstet Gynecol 2012; 55(04):864-874

2 Carapetis JR, Steer AC, Mulholland EK, Weber M. The global burden of group A streptococcal diseases. Lancet Infect Dis 2005;5(11):685-694

3 Centers for Disease Control and Prevention. 2016. Active Bacterial Core Surveillance Report, Emerging Infections Program Network, Group A Streptococcus 2016. Available at: https://www.cdc.gov/abcs/ reports-findings/survreports/gas16.html. Accessed December 10, 2019

4 Aronoff DM, Mulla ZD. Postpartum invasive group A streptococcal disease in the modern era. Infect Dis Obstet Gynecol 2008; 2008:796892

5 Deutscher M, Lewis M, Zell ER, Taylor TH Jr., Van Beneden C, Schrag S; Active Bacterial Core Surveillance Team. Incidence and severity of invasive Streptococcus pneumoniae, group A Streptococcus, and group B Streptococcus infections among pregnant and postpartum women. Clin Infect Dis 2011;53 (02):114-123

6 O'Loughlin RE, Roberson A, Cieslak PR, et al; Active Bacterial Core Surveillance Team. The epidemiology of invasive group A streptococcal infection and potential vaccine implications: United States, 2000-2004. Clin Infect Dis 2007;45(07):853-862

7 Anderson BL. Puerperal group A streptococcal infection: beyond Semmelweis. Obstet Gynecol 2014;123(04):874-882

8 Soper DE. Early recognition of serious infections in obstetrics and gynecology. Clin Obstet Gynecol 2012;55(04):858-863

9 Dellinger RP, Levy MM, Rhodes A, et al; Surviving Sepsis Campaign Guidelines Committee including the Pediatric Subgroup. Surviving sepsis campaign: international guidelines for management of severe sepsis and septic shock: 2012. Crit Care Med 2013;41(02): 580-637 